\title{
COMPARING THE TOXIC EFFECTS OF NONSTEROIDAL ANTI-INFLAMMATORY DRUGS (CELECOXIB AND IBUPROFEN) ON HEART, LIVER, AND KIDNEY IN RATS
}

\author{
NOOR D AZIZ ${ }^{1 *}$, MAZIN H OUDA ${ }^{2}$, MOAYAD MIJBIL UBAID ${ }^{3}$ \\ ${ }^{1}$ Department of Clinical Pharmacy, Faculty of Pharmacy, University of Kerbala, Iraq. ${ }^{2}$ Department of Pharmacology and Toxicology, Faculty \\ of Pharmacy, University of Kerbala, Iraq. ${ }^{3}$ Department of Biology, Basic Education College, University of Sumer, Iraq. \\ Email: noor.dheyaa@gmail.com \\ Received: 26 February 2018, Revised and Accepted: 18 April 2018
}

ABSTRACT

Objective: Nonsteroidal anti-inflammatory drugs (NSAIDs) do not reverse the disease progression, but they provide relief from pain and inflammation by inhibiting cyclooxygenase (COX) enzymes mediating the inflammatory pathway. Our aim was to make a meaningful comparison of both selective and non-selective COX-2 inhibitor to evaluate their toxic effects by measuring biochemical and histological alterations of heart, liver, and kidney.

Methods: This study was conducted on 18 Sprague-Dawley rats of both sexes for 30 days, rats were divided into three groups (control group, ibuprofen group, and celecoxib group) each group included six rats.

Results: The results are revealed that serum level of alanine aminotransferase, aspartate aminotransferase, alkaline phosphates, and total serum bilirubin was significantly increased $(\mathrm{p}<0.05)$ in ibuprofen and celecoxib group when compared with control, the highest level in celecoxib group, also serum level of urea was significantly elevated $(\mathrm{p}<0.05)$ in ibuprofen group when compared with control and celecoxib groups. Histopathological changes in cardiac tissue represented by vascular congestion and pericardial infiltration which are more prominent in celecoxib group, the changes in liver tissue revealed by vascular congestion and mild portal tract inflammation which is chronic in celecoxib group, while histological alterations in kidney tissue represented by severe vascular congestion with tubular necrosis which is more prominent in ibuprofen group.

Conclusion: Both ibuprofen and celecoxib group have toxic effects on heart, liver, and kidney represented by the biochemical and histopathological findings.

Keywords: Celecoxib, Ibuprofen, Toxicity, Biochemical markers, Histopathology, Heart, Liver, Kidney.

(C) 2018 The Authors. Published by Innovare Academic Sciences Pvt Ltd. This is an open access article under the CC BY license (http://creativecommons. org/licenses/by/4. 0/) DOI: http://dx.doi.org/10.22159/ajpcr.2018.v11i6.25487

\section{INTRODUCTION}

Nonsteroidal anti-inflammatory drugs (NSAIDs) are the most widely used drugs in management of pain, fever, redness, and edema arising as a consequence of inflammatory mediators release [1,2]. NSAIDs exert their anti-inflammatory, analgesic, and antipyretic effects by inhibiting of prostaglandins (PGs) synthesis, by suppressing the enzyme cyclooxygenase (COX), which are the enzymes that convert arachidonic acid into PGs, thromboxanes, and prostacyclins [2,3]. Two isoforms of COX have been recognized: Cox-1 and COX-2. The "COX-1" isoenzyme is usually presented in all tissues [4,5] and its stimulation leads to the formation of "PGs" essential in the maintenance of organ systems such as protecting the stomach wall and the kidney functions [6]. On the other hand, "COX-2" is always unexpressed in most tissues under normal physiological conditions $[7,8]$, but it is expressed when there is damaging in the body, leading to the induction of PGs synthesis [9].

Ibuprofen and celecoxib groups are "NSAIDs" mainly used for its antipyretic, anti-inflammatory, and analgesic properties [10]. The major mechanism of action of ibuprofen is the non-selective, reversible inhibition of the "COX enzymes COX-1 and COX-2" [11]. Ibuprofen is completely metabolized; the major route of elimination is oxidative metabolism by cytochrome p2C9 enzymes into inactive metabolites. Urinary excretion of the two major metabolites, "carboxy-ibuprofen" and "2-hydroxy-ibuprofen" represents $25 \%$ of the administered dose [12]. Celecoxib has also experimented in cancer prevention and has been used as an adjunct to surgery to limit the number of "adenomatous colorectal polyps" in patients with the genetic susceptibility for colon cancer syndrome, familial adenomatous polyposis $[13,14]$. Celecoxib is one of the subclasses of "NSAIDs," which were synthesized as "COX-2" selective inhibitors that are sometimes called coxibs $[15,16]$. Celecoxib expresses its anti-inflammatory and analgesic properties by blocking the synthesis of different inflammatory "prostanoids" [17]. However, the selectivity for COX-2 determined by in vitro is lower for celecoxib in comparison with other drugs in the coxib group (e.g., rofecoxib, valdecoxib, lumiracoxib, and etoricoxib), it is very similar at therapeutic concentration in vivo. Celecoxib also has the ability to suppress COX-1 compared with other coxibs; however, the result of this with regard to its therapeutic efficacy and toxicity are not well understood $[18,19]$. Celecoxib is mainly metabolized in the liver, with very little drug $<3 \%$ being excreted unchanged. The major routes of elimination for celecoxib are urine and feces. NSAIDs are in association with the incidence of serious adverse "cardiovascular thrombotic events" such as myocardial infarction and stroke. Risk may be associated with duration of use or pre-existing cardiovascular risk factors or diseases [20]. The objective of the study was to make a meaningful comparison of both selective and non-selective COX-2 inhibitor to evaluate their toxic effects by measuring biochemical and histological alterations of heart, liver, and kidney.

\section{METHODS}

Experimental animals

This study was conducted on 18 Sprague-Dawley rats of both sexes weighing between 250 and $300 \mathrm{~g}$ were used in this research. They were separated into three groups each group consists of six animals maintained in animal house of University of Kerbala/College of Pharmacy with free access to food and water ad libitum. As the following:

1- Control group: Drenched normal saline for 1 month.

2- Ibuprofen group: Drenched $40 \mathrm{mg} / \mathrm{kg} /$ day of ibuprofen for 1 month.

3- Celecoxib group: Drenched $40 \mathrm{mg} / \mathrm{kg} /$ day of celecoxib for 1 month. 
The study was conducted after obtaining approval from the Ethics Committee of College of Pharmacy/University of Kerbala.

\section{Experimental technique}

Drugs used including ibuprofen syrup $100 \mathrm{mg} / 5 \mathrm{ml}$ (Philadelphia, Jordan), at $40 \mathrm{mg} / \mathrm{kg}$; celecoxib capsule $200 \mathrm{mg}$ (Pfizer, Switzerland), at $40 \mathrm{mg} / \mathrm{kg}$. Celecoxib was dissolved in distilled water before administration to each animal in the group orally using a stomach cannula for 30 days. The animals were observed in their cages for clinical symptoms daily. At the end of the experimental period, the animals were anesthetized using chloroform and blood collected by cardiac puncture for serum biochemical analysis

\section{Determination of biochemical parameters}

Serum was separated from clotted blood obtained by cardiac puncture. Serum enzymes alanine aminotransferase (ALT), aspartate aminotransferase (AST), alkaline phosphates (ALP), and total serum bilirubin (TSB) were determined by procedures of Sigma diagnostics, serum creatinine, and blood urea nitrogen by method of Crocker.

\section{Preparation of histopathological slides}

Organs such as the heart, liver, and kidney were isolated into $10 \%$ saline formalin and then subjected to histological procedures and preparation of tissue slides as directed by Banchroft et al. [21].

\section{Statistical analysis}

The results were represented as mean \pm standard error mean. Differences between control and other experimental groups were tested for statistical significance using SPSS version (20) one-way analysis of variances. Statistically significant differences exist at $p \leq 0.05$.

\section{RESULTS}

\section{Clinical effects of NSAIDs in rats}

Animals administered with ibuprofen showed reduce food intake, sluggishness, and diarrhea with some mortality. The only symptoms seen in the celecoxib group was sluggishness and some mortality.

\section{Effect of the NSAIDs on the serum level of ALT, AST, ALP, and TSB in rats}

The results showed significant increase $(p<0.05)$ in serum level of AST, ALT, ALP, and TSB in celecoxib group when compared with ibuprofen and control groups, while for ibuprofen group the results revealed significant increase $(p<0.05)$ in serum level of AST and TSB, with insignificant difference $(p>0.05)$ in the level of ALT and ALP when compared with control group (Table 1)

Effect of the NSAIDs on the serum level of urea and creatinine in rats The results showed significant increase $(\mathrm{p}<0.05)$ in serum level of urea $(29.66,41.50$, and 35.83$)$ with the highest level in the ibuprofen group when compared with both celecoxib and control groups, also there is insignificant difference $(p>0.05)$ in the serum level of creatinine $(0.69$, 0.77 , and 0.72 ) between the three groups (Table 1 ).

As shown in Fig. 1a, the histological features of the normal heart indicated a normal looking endocardium and myocardium including cardiac muscle and fibers. In heart sections of ibuprofen-treated group, clarifying preserved architecture with prominent vascular congestion and mild pericardial infiltration by chronic inflammatory cell and macrophages (Fig. 1b). In comparison with heart sections of celecoxib treated group clarifying more prominent vascular congestion with pericardial acute inflammatory cell infiltration (feature of pericarditis) (Fig. 1c)

As shown in Fig. 2a, the histological features of the normal liver indicated a normal liver clarifying preserved architecture of liver lobular and acini including central vein and portal tract. In comparison with liver sections of ibuprofen treated group, clarifying vascular congestion within central venula with mild portal tract inflammation (Fig. 2b). In celecoxib pretreated group, clarifying preserved architecture vascular congestion within central venula associated with mild chronic portal tract inflammation (Fig. 2c)

As shown in Fig. 3a, the histological features of the normal kidney indicated a normal kidney clarifying the normal architecture of cortex and medulla, normal looking glomeruli and tubules. In comparison with kidney sections of ibuprofen treated group, clarifying vascular congestion of both cortex and medulla, with partial tubular necrosis (Fig. 3b). In celecoxib pretreated group, clarifying preserved architecture, severe and diffuse vascular congestion not confined mainly in the cortex. There is focal increase in the mesangium of some glomeruli (fibrosis within glomeruli) medulla and tubules are unremarkable apart from some clearing changes (focal in the medulla mainly) (Fig. 3c).

\section{DISCUSSION}

According to the present study, we observed the administration of both celecoxib and Ibuprofen group resulted in the induction of biochemical and histopathological abnormalities of cardiac, hepatic, and renal tissues of the rats. Histologically, the cardiac manifestations are vascular congestion and mild pericardial infiltration by chronic inflammatory cell and macrophages which is more prominent in celecoxib group with feature of pericarditis, this result is in agreement with (Sclott et al., 2005) cardiovascular risk with celecoxib to prevent colorectal adenomas led to a dose-related increase in the risk of serious cardiovascular events, including death from cardiovascular causes, myocardial infarction, stroke, and heart failure [22].

Histologically, the hepatic alterations are manifested by vascular congestion within central venula and mild portal tract inflammation which is chronic in celecoxib group, also serum level reflecting significant alterations observed in the biochemical indices of rats showed that administration of celecoxib at the given doses may cause hepatotoxicity. These results are in agreement with Nachimuthu et al. (2001) observation of celecoxib in clinical trials [23]. The activities of AST, ALT, and ALP are commonly used as biochemical indicators of liver functions. Structural and functional alterations in the liver result in elevated levels of these enzymes in the circulation. The levels of these aminotransferases (ALT and AST) in serum are elevated in all liver diseases. In fact, very high levels of more than 1000 units can be seen in acute hepatitis [24]

In our present study, we clearly demonstrate that both ibuprofen and celecoxib administration lead to histological alterations in

Table 1: The effect of ibuprofen and celecoxib on the serum levels of ALT, AST, urea, creatinine, ALP, and TSB in comparison with control group

\begin{tabular}{|c|c|c|c|c|c|c|}
\hline \multirow[t]{3}{*}{ Groups } & \multicolumn{6}{|l|}{ Parameters } \\
\hline & \multicolumn{6}{|l|}{ Mean \pm SEM } \\
\hline & $\operatorname{ALT}(\mathrm{U} / \mathrm{L})$ & AST (U/L) & Urea (mg/dl) & Creatinine $(\mathrm{mg} / \mathrm{dl})$ & $\operatorname{ALP}(U / L)$ & TSB (mg/dl) \\
\hline Control & $35.16 \pm 1.44$ & $64.83 \pm 3.28$ & $29.66 \pm 1.83$ & $0.69 \pm 0.03$ & $280.33 \pm 27.85$ & $0.28 \pm 0.02$ \\
\hline Ibuprofen & $39.33 \pm 2.24$ & $85.33^{*} \pm 2.62$ & $41.5^{*} \pm 1.64$ & $0.77 \pm 0.02$ & $346.00 \pm 31.78$ & $0.44^{*} \pm 0.02$ \\
\hline Celecoxib & $51.83^{*} \pm 2.98$ & $119.33^{*} \pm 8.46$ & $35.8^{*} \pm 1.35$ & $0.72 \pm 0.04$ & $512.16^{*} \pm 51.41$ & $0.52^{*} \pm 0.02$ \\
\hline
\end{tabular}

*The mean difference is significant at the 0.05 level. SEM: Standard error mean, ALT: Alanine aminotransferase, AST: Aspartate aminotransferase, ALP: Alkaline phosphates, TSB: Total serum bilirubin 


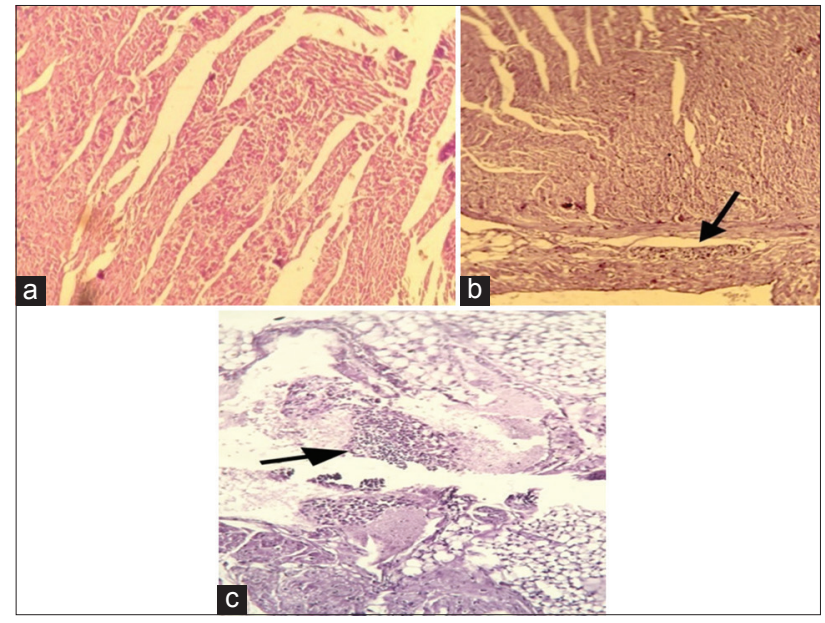

Fig. 1: Histological sections of cardiac tissues from untreated and treated groups. (a) Histology of normal control rat heart $(\times 400)$; (b) Mild Pericardial infiltration - histopathological changes seen in rats treated with ibuprofen; (c) Prominent vascular congestion and pericarditis - heart of rats pretreated with celecoxib

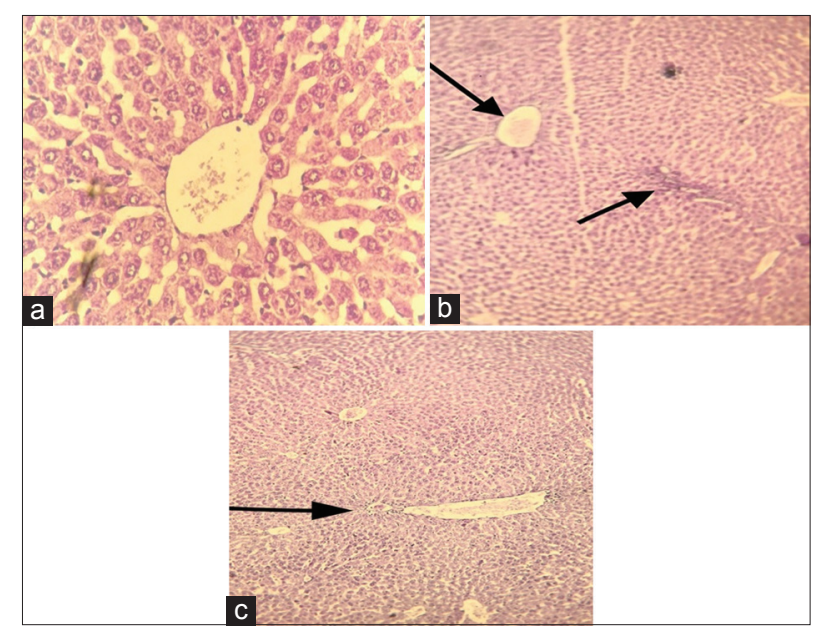

Fig. 2: Histological sections of livers from untreated and treated groups. (a) Histology of normal control rat liver $(\times 400)$;

(b) vascular Congestion and portal tract - histopathological changes seen in rats treated with ibuprofen; (c) vascular Congestion chronic inflammation - liver of rats pretreated with celecoxib

kidney represented by severe vascular congestion of both cortex and medulla, with partial tubular necrosis in ibuprofen group; on the other hand, celecoxib group showed severe and diffuse vascular congestion not confined mainly in the cortex. There is focal increase in the mesangium of some glomeruli (fibrosis within glomeruli), and partial tubular necrosis, the present findings are in agreement with the work of Rania et al. (2013) demonstrate that at higher doses chronic NSAIDs contribute to the onset of glomerular changes in the filtration barrier [25]. Celecoxib resulted in glomeruli basement membrane thinning, slit pore diameter and foot process, density reduction, and increased mesangial area, but at comparable doses, ibuprofen is more detrimental than celecoxib, causing severe necrotizing pyelonephritis.

\section{CONCLUSION}

NSAIDs are the mainstay therapy in controlling of pain and fever by inhibiting COX enzymes mediated inflammatory response so that the chronic use of these drugs makes the patients exposed to adverse and toxic effects of it, and by making comparison of both selective (celecoxib) and non-selective COX-2 inhibitor (ibuprofen) to evaluate

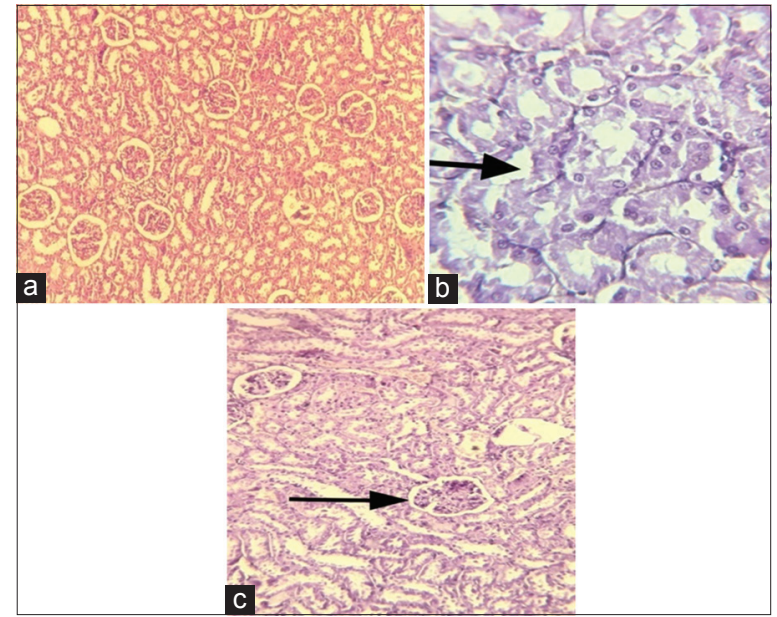

Fig. 3: Histological sections of kidneys from untreated and treated groups. (a) Histology of normal control rat kidney $(\times 400)$; (b) Partial tubular necrosis - histopathological changes seen in rats treated with ibuprofen; (c) Increase in the mesangium of glomeruli - kidney of rat pretreated with celecoxib

their toxic effects by measuring biochemical and histological alterations which showed significant findings for heart, liver, and kidney.

\section{ACKNOWLEDGMENT}

Special gratitude is expressed to the department of pharmacology and toxicology that are represented by the headmaster and the staff for providing facilities required for this research, and all who participates in this work

\section{AUTHOR'S CONTRIBUTIONS}

Noor D. Aziz contributed to the idea of the research, write, and coordinate the work between the other members. Mazi H. Ouda contributed to drug dosing to the rats and follow-up of the animal for 30 days (the research period). Moayad Mijbil Ubaid contributed to biostatistics of the research.

\section{CONFLICTS OF INTEREST}

There are no conflicts of interest.

\section{REFERENCES}

1. Ferreira S. Peripheral analgesic sites of action of anti-inflammatory drugs. Int J Clin Pract Suppl 2002;128:2-10.

2. Mitchell JA, Warner TD. Cyclo-oxygenase-2: Pharmacology, physiology, biochemistry and relevance to NSAID therapy. Br J Pharm 1999;128:1121-32.

3. Brune K. Persistence of NSAIDs at effectsites and rapid disappearance from side-effect compartments contributes to tolerability. Curr Med Res Opin 2007;23:2985-95.

4. Hla T, Neilson K. Human cyclooxygenase-2 cDNA. Proc Natl Acad Sci 1992;89:7384-8.

5. Peniva PT. Non-steroidal anti-inflamatory dugs for topical ophthalmic administration: Contemporary trends. Int J Pharm Pharm Sci 2015;7:13-9.

6. Scarpignato C, Corradi C, Gandolfi MA, Galmiche JP. A new technique for continuous measurement and recording of gastric potential difference in the rat: Evaluation of NSAID-induced gastrics mucosal damage. J Pharm Toxicol Methods 1995;34:63-72.

7. Famaey J. In vitro and in vivo pharmacological evidence of selective cyclooxygenase- 2 inhibition by nimesulide: An overview. Inflamm Res 1997;46:437-46.

8. Miladiyah I, Jumina J, Haryana SM, Mustofa M. In silico molecular docking of xanthone derivatives as cyclooxygenase-2 inhibitor agents. Int J Pharm Pharm Sci 2017;9:98-104.

9. Smith WL, Meade EA, DeWitt DL. Interactions of PGH synthase isozymes-1 and-2 with NSAIDs. Ann N Y Acad Sci 1994;744:50-7.

10. Rainsford K. Ibuprofen pharmacology, efficacy and safety. Inflamm 
Pharmacol 2009; 17:275-342

11. Prusakiewicz JJ, Duggan KC, Rouzer CA, Marnett LJ. Differential sensitivity and mechanism of inhibition of COX-2 oxygenation of arachidonic acid and 2-arachidonoylglycerol by ibuprofen and mefenamic acid. Biochemistry 2009;48:7353-5.

12. Rudy AC, Knight PM, Brater DC, Hall SD. Stereoselective metabolism of ibuprofen in humans: Administration of R, S-and racemic ibuprofen. J Pharm Exp Ther 1991;259:1133-9.

13. Bertagnolli MM, Eagle CJ, Zauber AG, Redston M, Solomon SD, Kim K, et al. Celecoxib for the prevention of sporadic colorectal adenomas. N Engl J Med 2006;355:873-84.

14. Steinbach G, Lynch PM, Phillips RK, Wallace MH, Hawk E, Gordon GB, et al. The effect of celecoxib, a cyclooxygenase-2 inhibitor, in familial adenomatous polyposis. N Engl J Med 2000;342:1946-52.

15. Lanas A. Clinical experience with cyclooxygenase-2 inhibitors. Rheumatology 2002;41 suppl_1:16-22.

16. Lanas A, Sopeña F. Nonsteroidal anti-inflammatory drugs and lower gastrointestinal complications. Gastroenterol Clin North Am 2009;38:333-52.

17. García-Alonso V, Titos E, Alcaraz-Quiles J, Rius B, Lopategi A, López-Vicario $\mathrm{C}$, et al. Prostaglandin E2 exerts multiple regulatory actions on human obese adipose tissue remodeling, inflammation, adaptive thermogenesis and lipolysis. PLoS One 2016;11:e0153751.

18. Levitt RJ, Buckley J, Blouin MJ, Schaub B, Triche TJ, Pollak M.
Growth inhibition of breast epithelial cells by celecoxib is associated with upregulation of insulin-like growth factor binding protein-3 expression. Biochem Biophys Res Commun 2004;316:421-8

19. Schönthal A. Direct non-cyclooxygenase-2 targets of celecoxib and their potential relevance for cancer therapy. $\mathrm{Br} \mathrm{J}$ Cancer 2007;97:1465-8

20. Gerald GA. Prostaglandins modulators of inflammation and cardiovascular risk. J Clin Rheumatol 2004;10:S12-S7.

21. Banchroft JD, Stevens A, Turner DR. Theory and Practice of Histological Techniques. $4^{\text {th }}$ ed. New York, London, San Francisco, Tokyo: Churchillliving Stone; 1996.

22. Solomon SD, McMurray JJ, Pfeffer MA, Wittes J, Fowler R, Finn P, et al. Cardiovascular risk associated with celecoxib in a clinical trial for colorectal adenoma prevention. N Engl J Med 2005;352:1071-80.

23. Nachimuthu S, Volfinzon L, Gopal L. Acute hepatocellular and cholestatic injury in a patient taking celecoxib. Postgrad Med J 2001;77:548-50

24. Vasudevan DM, Sreekumari S. Textbook of Biochemistry for Medical Students. $5^{\text {th }}$ ed. New Delhi: Jaypee BrothersMedical Publishers Ltd.; 2007.

25. Nasrallah R, Robertson SJ, Karsh J, H'ebert RL. Celecoxib modifies glomerular basement membrane, mesangium and podocytes in OVE26mice, but ibuprofen is more detrimental. Clin Sci $2013 ; 124: 685-94$. 